\title{
ZUR CHARAKTERISIERUNG DER ALGEBRAISCHEN UND DER RATIONALEN FUNKTIONEN DURCH IHRE FUNKTIONSWERTE.
}

\author{
VON \\ THEODOR SCHNEIDER
}

in GötTINGEN.

In dieser Note soll keine Charakterisierung der algebraischen bzw. rationalen Funktionen schlechthin versucht werden, sondern nur der algebraischen bzw. rationalen Funktionen in $z$, die einer Definitionsgleichnng mit algebraischen Zahlkoeffizienten genügen, woraus sich in bekannter Weise eine Beziehung mit ganzen rationalen Zahlkoeffizienten ergibt.

Lange ist vergeblich versucht worden, für die Algebraizität bzw. Rationalität einer Funktion charakteristische Bedingungen aus dem Wertevorrat aufzustellen, und es ist auch seit langem bekannt, dass selbst so umfassende Bedingungen wie z. B., dass die Funktion an allen algebraischen Stellen algebraische Funktionswerte annehme, nicht binreichend sind.

Erst kürzlich gelang K. Dörge ${ }^{1}$ die Angabe charakteristischer Bedingungen für algebraische und auch für rationale Funktionen durch den Wertevorrat, zusammen mit Eigenschaften einer Potenzreihenentwicklung.

Hier soll von einer einerseits wesentlich schwächeren Bedingung, die sich nur auf den Wertevorrat bezieht, bezüglich der einzelnen Funktionswerte weniger als bei Dörge verlangt, und von der Potenzreihe gar keinen Gebrauch macht, die allerdings andererseits über den Wertevorrat insofern mehr fordert, als sie über unendlich viele Funktionswerte aussagt, während Dörge mit einer hinreichend grossen Anzahl von Funktionswerten auskommt, gezeigt werden, dass sie für die Algebraizität charakteristisch ist. Fine gewisse Verschärfung dieser Bedingung wird die rationalen Funktionen kennzeichnen.

Aus dem Beweis wird leicht erkennbar sein, dass sich auf analoge Weise beliebig viele verschiedene solcher charakteristischer Bedingungen angeben lassen.

1 Die Arbeit von $K$. Dörge soll in den Mathematischen Annalen Bd 122 , Heft 2 erscheinen. Die Resultate derselben sind ron K. Dörge anf der Tagung der D. M. V. I949 vorgetragen worden. 
Wir beschränken uns hier auf ein bezüglich der Stellen möglichst einfaches Kriterium. Ohne Einschränkung der Allgemeinheit kann angenommen werden, dass die zu betrachtende Funktion $g(z)$ an der Stelle $z=0$ reguläres Verhalten besitzt. Sollte das nämlich nicht der Fall sein, so besitzt $g(z)$ sicher an einer Stelle $z_{0}$ reguläres Verhalten, wobei $z_{0}$ eine algebraische Zahl sei. Dann betrachte man statt $g(z)$ die Funktion $g\left(z-z_{0}\right)$, die sicher gleichzeitig mit $g(z)$ algebraisch oder rational ist. $g(z)$ darf dabei selbstverständlich mehrdeutig sein. Nun lautet:

Satz I (I. Kriterium für Algebraizität):

$g(z)$ ist dann und nur dann algebraisch in $z$, wenn

a) die Funktionswerte von $g(z)$ auf einem Blatt der Riemannschen Fläche von $g(z)$ an den Stellen $z=\frac{1}{v}$ für alle natürlichen Zạhlen $v$ derart, dass diese Stellen $\frac{\mathrm{I}}{\mathrm{v}}$ in einer festen Umgebung von $z=0$ liegen, algebraische Zahlen höchstens s-ten Grades sind, wobei s eine feste natürliche Zahl bedeute, und

b)

$$
\varlimsup_{\nu \rightarrow \infty} \frac{\log \log H\left(g\left(\frac{\mathrm{I}}{\nu}\right)\right)}{\log \nu}<\mathrm{I}
$$

ist, wobei $H\left(g\left(\frac{\mathrm{I}}{\nu}\right)\right)$ die Höhe von $g\left(\frac{\mathrm{I}}{\nu}\right)$, d.h. das Maximum der Beträge der ganzen rationalen, teilerfremden Koeffizienten in der irreduziblen Gleichung für $g\left(\frac{1}{v}\right)$ bedeute.

Durch Verschärfung der Forderung a) entsteht hieraus

Satz II (r. Kriterium für Rationalität):

$g(z)$ ist dann und nur dann rational in $z$, wenn

a) die Funktionswerte von $g(z)$ auf einem Blatt der Riemannschen Fläche von $g(z)$ an allen Stellen $z=\frac{1}{v} ; \boldsymbol{v}=1,2, \ldots$, die in einer festen Umgebung von $z=0$ liegen, einem festen algebraischen Zahlkörper angehören, und

b)

$$
\varlimsup_{\nu \rightarrow \infty} \frac{\log \log H\left(g\left(\frac{\mathrm{I}}{v}\right)\right)}{\log v}<\mathrm{I}
$$

ist. 
Es ist leicht einzusehen, wie sich die genannten Kriterien auf Kriterien für algebraische, bzw. rationale Abhängigkeit einer Funktion von einer anderen Funktion übertragen lassen. Ferner gelingt es unschwer, die aufgeführten Sätze auf Funktionen mehrerer Veränderlicher auszudehnen.

\section{Beweise:}

Die Notwendigkeit der angegebenen Bedingungen ist recht einfach zu erkennen. Ist nämlich $g(z)$ eine algebraische, bzw. rationale Funktion in $z$ mit algebraischen Koeffizienten, die für $z=0$ endlich ist, so sind die Eigenschaften a) von Satz I bzw. Satz II offenbar erfüllt. Um die Notwendigkeit der Aussage b) der beiden Sätze einzusehen, mache man sich klar, dass $g\left(\frac{I}{\nu}\right)$ eine algebraische Zahl vom Grade $\sigma \leq s$ ist, die als Wurzel einer algebraischen Gleichung mit ganzalgebraischen Koeffizienten dargestellt ist. Diese Koeffizienten sind Polynome in $v$ von beschränktem Grade mit festen ganzalgebraischen Zahlkoeffizienten, entstanden durch Einsetzen von $z=\frac{1}{v}$ in der algebraischen Definitionsgleichung für $g(z)$ :

$$
A(z, g(z))=\sum_{i, x=0}^{i, k} a_{\iota x} z^{\iota}(g(z))^{x}
$$

und Multiplikation derselben mit $\nu^{i}$ und dem ganzrationalen Hauptnenner der algebraischen Zahlen $a_{\iota x}(\iota=0, \ldots, i ; x=0, \ldots, k)$. Analog der so aus (I) hervorgegangenen Gleichung mit ganzalgebraischen Koeffizienten bilde man für $g\left(\frac{I}{v}\right)$ durch Ersetzen der algebraischen Koeffizienten durch deren Konjugierte die sämtlichen, bezüglich des Körpers der rationalen Zahlen, konjugierte Gleichungen. Das Produkt derselben, zusammen mit obiger Gleichung für $g\left(\frac{I}{\nu}\right)$, ergibt dann eine algebraische, möglicherweise reduzible Gleichung für $g\left(\frac{I}{\nu}\right)$ mit ganzen rationalen Koeffizienten, deren Höhe als Funktion von $v$ höchstens wie eine feste Potenz von $\nu$ wächst. Dann wächst aber die Höhe der irreduziblen Gleichung für $g\left(\frac{I}{\nu}\right)$ mit teilerfremden, ganzen rationalen Koeffizienten, wie leicht einzusehen ist (siehe z. B. in ${ }^{1}$ ), ebenfalls wie eine feste Potenz von $\nu$. Es ist also

${ }^{1}$ C. L. SIEGEL, Approximation algebraischer Zahlen, Mathematische Zeitschrift 10 (1921), 173-213, insbesondere Hilfssatz JII, S. 176. 
und daraus folgt,

$$
\varlimsup_{\nu \rightarrow \infty} \frac{\log H\left(g\left(\frac{\mathrm{I}}{\nu}\right)\right)}{\log \nu}<t,
$$

$$
\lim _{\nu \rightarrow \infty} \frac{\log \log H\left(g\left(\frac{\mathrm{I}}{\nu}\right)\right)}{\log v}=0,
$$

also erst recht das Erfülltsein von Bedingung b).

Nicht ganz so einfach ist der Nachweis, dass die in den Sätzen I und II aufgeführten Bedingungen auch hinreichend sind.

Zuerst soll der Beweis von Satz I zu Fnde geführt werden, d.h. es soll noch gezeigt werden, dass die Bedingungen a) und b) für eine bei $z=0$ reguläre einoder mehrdeutige Funktion auch hinreichend sind.

Ist $g(z)$ eine analytische Funktion, die in der Umgebung von $z=0$ keine Singularität aufweist, so ist $g(z)$ insbesondere auf dem in Satz I betrachteten Blatt der Riemannschen Fläche von $g(z)$ in $|z|<r$ regulär. Wählt man $v_{0}=\mathrm{I}+\left[\frac{2}{r}\right]$, so ist sogar $\frac{1}{v}<\frac{r}{2}$ für alle $\nu>v_{0}$, also $g\left(\frac{1}{v}\right)$ endlich und nach den Voraussetzungen algebraisch von höchstens $s$-tem Grade.

Man setze nun ein Polynom

$$
\phi(z)=\sum_{\lambda, \mu=0}^{l, m} c_{\lambda \mu} z^{\lambda}(g(z))^{\mu}
$$

mit unbestimmten Koeffizienten $c_{\lambda \mu}$ an, und bestimme die $c_{\lambda \mu}$ als nicht sämtlich verschwindende, ganze rationale Zahlen so, dass $\phi(z)$ auf dem in Satz I betrachteten Blatt der Riemannschen Fläche von $g(z)$ in der Umgebung des Nullpunktes für alle Werte $z=\frac{I}{\nu}, v$ ganzzahlig und gleich $\nu_{0}+1, \ldots, \nu_{0}+n$, verschwindet, wobei über $l, m, n$ noch Festsetzungen zu treffen sind.

Jede Forderung $\phi\left(\frac{1}{v}\right)=0$ bedeutet eine lineare homogene Gleichung für die $c_{\lambda \mu}$ mit algebraischen Koeffizienten aus einem Körper höchstens $s$-ten Grades, und diese zerfällt, da die $c_{\lambda \mu}$ ganzrational sein sollen, vermittels der Basisdarstellung der algebraischen Koeffizienten, in höchstens $s$ lineare homogene Gleichungen für die $c_{\lambda \mu}$ mit rationalen Koeffizienten. Es kommt darauf an, Zähler und Nenner dieser rationalen Koeffizienten abzuschätzen. 
Sei mit der für diesen Zweck einzuführenden abkürzenden Bezeichnung $g\left(\frac{\mathrm{I}}{\nu}\right)=\beta_{\nu}=\beta$ bei festem $\nu$, so wird die aus $\phi\left(\frac{\mathrm{I}}{\nu}\right)=0$ entstandene homogene lineare Gleichung für die $c_{\lambda \mu}$ nach Multiplikation mit $\nu^{l}$

$$
\sum_{\lambda, \mu=0}^{l, m} \nu^{l-\lambda} \beta^{\mu} c_{\lambda \mu}=0
$$

Da $\beta_{v}$ nach Voraussetzung algebraisch vom Grade $\sigma \leq s$ ist, lautet das Minimalpolynom für $\beta_{v}$ mit teilerfremden, ganzen rationalen Koeffizienten

mit

$$
\sum_{x=0}^{\sigma} b_{x} \beta^{x}=0
$$

$$
\underset{x=0, \ldots, \sigma}{\operatorname{Max}}\left|b_{x}\right|=H\left(g\left(\frac{\mathrm{I}}{\nu}\right)\right)
$$

und damit erhält man durch Induktion nach $\mu$ die Darstellung

$$
\beta^{\mu}=b_{\sigma}^{-\mu}\left(d_{0, \mu}+d_{1, \mu} \beta+\cdots+d_{\sigma-1, \mu} \beta^{\sigma-1}\right)
$$

mit ganzen rationalen

$$
d_{0, \mu}, \ldots, d_{\sigma-1, \mu} ; \mu \geq 0,
$$

und es folgt ebenfalls durch Induktion nach $\mu$ die Abschätzung

$$
\left|d_{x, \mu}\right| \leq\left\{2 H\left(g\left(\frac{\mathrm{I}}{\nu}\right)\right)\right\}^{\mu} \quad(x=0, \ldots, \sigma-\mathrm{I}) .
$$

Wegen der linearen Unabhängigkeit von $1, \beta, \ldots, \beta^{\sigma-1}$ im Körper der rationalen Zahlen zerfällt die objge Gleichung für die rationalen Unbestimmten $c_{\lambda \mu}$ in die $\sigma$ linearen homogenen Gleichungen mit rationalen Koeffizienten

oder

$$
\sum_{\lambda, \mu=0}^{l, m} v^{l-\lambda} b_{\sigma}^{-\mu} d_{x, \mu} \beta^{x} c_{\lambda \mu}=0 \quad(x=0, \ldots, \sigma-1)
$$

$$
\sum_{\lambda, \mu=0}^{l, m} \nu^{l-\lambda} b_{\sigma}^{m-\mu} d_{x, \mu} c_{\lambda \mu}=0
$$

mit ganzen rationalen Koeffizienten, deren Beträge

$$
\left|b_{\sigma}^{m-\mu} d_{x, \mu} \nu^{l-\lambda}\right| \leq 2^{m}\left\{H\left(g\left(\frac{1}{y}\right)\right)\right\}^{2 m} v^{l} \quad(x=0, \ldots, \sigma-1)
$$

sind. Damit haben wir für beliebiges $v$ jede der linearen Gleichungen für die $c_{\lambda \mu}$ mit algebraischen Koeffizienten auf höchstens $s$ lineare homogene Gleichungen 
mit ganzen rationalen Koeffizienten, für die absolut genommen bei Berücksichtigung von Voraussetzung b)

gilt, zurückgeführt.

$$
\leq 2^{m} e^{2 m v} \nu^{l}=O\left(\nu^{l} e^{3 m v}\right)
$$

Die Forderung, dass $\phi(z)$ für $z=\frac{\mathrm{I}}{\nu_{0}+\mathrm{I}}, \cdots, z=\frac{\mathrm{I}}{\nu_{0}+n}$ verschwinde, bedentet demnach für die $c_{\lambda \mu}$ das Bestehen von höchstens $s \cdot n$ linearen homogenen Gleichungen mit ganzrationalen Koeffizienten, die höchstens von der Grössenordnung $O\left(\left(\nu_{0}+n\right)^{l} e^{3 m\left(v_{0}+n\right)}\right)$ sind. Die Anzahl der Unbestimmten beträgt $(l+\mathrm{I})(m+\mathrm{I})$, und für diese Anzahl gelte

$$
(l+\mathrm{I})(m+\mathrm{I}) \geq 2 s n
$$

Es werde nun

(3)

$$
m=\left[\log ^{\frac{1}{2}} n\right]
$$

und

(4)

$$
l=\left[2 \sin \log ^{-\frac{1}{2}} n\right]
$$

gesetzt.

Dann ist (2) erfüllt, und die ganzrationalen Koeffizienten der Gleichungen für die $c_{\lambda \mu}$ sind nun höchstens von der Ordnung

$$
O\left(e^{28 n \log \frac{1}{2} n+4 n \log \frac{1}{2} n}\right)=O\left(e^{(2 s+4) n \log \frac{1}{2} n}\right)
$$

als Funktion von $n$. Ein bekannter Satz ${ }^{1}$, der sich mittels des Schubfachschlusses leicht zeigen lässt, besagt, dass das lineare homogene Gleichungssystem in nicht sämtlich verschwindenden, ganzen rationalen $c_{\lambda \mu}$, für die

(5)

$$
\left|c_{\lambda \mu}\right|=O\left(e^{(2 s+1) n \log \frac{1}{2} n}\right)
$$

gilt, lösbar ist.

Nun soll gezeigt werden: Verschwindet $\phi(z)$ für $z=\frac{\mathrm{I}}{v_{0}+\mathrm{I}}, \cdots: z=\frac{\mathrm{I}}{\nu_{0}+n_{1}}$ auf einem Blatt der Riemannschen Fläche, so auch für $z=\frac{I}{v_{0}+n_{1}+\text { I }}$ auf dem gleichen Blatt, wenn nur $n_{1} \geq n$ und $n$ hinreichend gross, aber gegen $n_{1}$ fest ist.

Der Beweis dieses Hilfssatzes lässt sich auf verschiedene Weise führen. Hier soll das Maximumprinzip herangezogen werden.

${ }^{1}$ Siehe z. B. C. I. SIEgEL, Über einige Anwendungen diophantischer Approximationen, Abb. Preuss. Akad. Wiss., Berlin, I930. 
Sei $\Gamma$ ein Kreis um den Nullpunkt mit dem Radius $R=\frac{3}{4} r$. Da dieser Kreis die Stellen $z=\frac{1}{v_{0}+\mathrm{I}}, \ldots, z=\frac{\mathrm{I}}{v_{0}+n_{1}}$ sämtlich im Innern enthält, ist die Funktion

$$
{\frac{\mathrm{I}}{\left(z-\frac{\mathrm{I}}{v_{0}+\mathrm{I}}\right)} \cdots\left(z-\frac{\mathrm{I}}{v_{0}+n_{1}}\right)}^{\phi(z)}
$$

im Innern der Kurve $\Gamma$ regulär. Nach dem Maximumprinzip liegt daher das Maximum der Beträge der Werte dieser Funktion auf dem betrachteten Blatt der Riemannschen Fläche für alle Stellen $z$ mit $|z| \leq R$ auf dem Rande des Kreises $\Gamma$. Daher gilt die Ungleichung

$$
\begin{aligned}
& \left|\frac{\phi\left(\frac{\mathrm{I}}{v_{0}+n_{1}+\mathrm{I}}\right)}{\left(\frac{\mathrm{I}}{v_{0}+n_{1}+\mathrm{I}}-\frac{\mathrm{I}}{v_{0}+\mathrm{I}}\right) \cdots\left(\frac{\mathrm{I}}{v_{0}+n_{1}+\mathrm{I}}-\frac{\mathrm{I}}{v_{0}+n_{1}}\right)}\right| \leq \\
& \quad \leq \operatorname{Max}_{|\neq|=R}\left(\mid \frac{\phi(z)}{\left(z-\frac{\mathrm{I}}{v_{0}+\mathrm{I}}\right) \cdots\left(z-\frac{\mathrm{I}}{v_{0}+n_{1}}\right)}\right)
\end{aligned}
$$

Hieraus werde $\left|\phi\left(\frac{\mathrm{I}}{\nu_{0}+n_{1}+\mathrm{I}}\right)\right|$ abgeschätzt.

Da $r$ fest ist, ergibt sich die rechte Seite von (6) unter Berücksichtigung von (5) und $n \leq n_{1}$ als Funktion von $n_{1}$ von der Ordnung

$$
O\left(e^{(2 s+4) n_{1} \log n_{n_{1}}} \cdot 2^{l} \cdot \gamma_{1}^{m} \cdot\left(\frac{r}{4}\right)^{-n_{1}}\right)
$$

mit einer von $n_{1}$ unabhängigen Konstanten $\gamma_{1}$. Daraus folgt bei Beachtung von (3) und (4), dass die rechte Seite von (6) die Grössenordnung

besitzt. Schliesslich ist

$$
O\left(e^{(2 s+5) n_{1} \log s_{1}}\right)
$$

$$
\begin{aligned}
&\left|\left(\frac{\mathrm{I}}{\nu_{0}+n_{1}+\mathrm{I}}-\frac{\mathrm{I}}{\nu_{0}+\mathrm{I}}\right) \cdots\left(\frac{\mathrm{I}}{\nu_{0}+n_{1}+\mathrm{I}}-\frac{\mathrm{I}}{\nu_{0}+n_{1}}\right)\right| \leq \\
& \leq \frac{\mathrm{I}}{\left(\nu_{0}+\mathrm{I}\right) \ldots\left(v_{0}+n_{1}\right)}=\frac{\nu_{0} !}{\left(v_{0}+n_{1}\right) !},
\end{aligned}
$$

also, da $\nu_{0}$ fest ist, $\leq \gamma_{2}^{n_{1}} n_{1}^{-n_{1}}$, wobei wieder $\gamma_{2}$ eine von $n_{1}$ unabhängige Konstante bedeute. Damit erhalten wir 


$$
\left|\phi\left(\frac{\mathrm{I}}{v_{0}+n_{1}+1}\right)\right|=O\left(e^{(2 s+6) n_{1} \log \frac{1}{2} n_{1}-n_{1} \log n_{1}}\right) .
$$

Diese Zahl $\phi\left(\frac{1}{\nu_{0}+n_{1}+\mathrm{I}}\right)$ ist nach Voraussetzung und der Bestimmung von $\phi(z)$ algebraisch von einem Grade $\sigma \leq s$, und ihr Produkt mit einer nicht verschwindenden, ganzen rationalen Zahl $Q$, deren Grösse ebenfalls aus der Bildung von $\phi(z)$ sofort zu entnehmen ist, ist ganzalgebraisch. Für $Q$ findet man wegen $n_{1} \geq n$

$$
|Q|=O\left(e^{(28+2) n_{1} \log \frac{1}{t_{1}} n_{1}}\right)
$$

Für die höchstens $s$ Konjugierten $\phi^{(\tau)}\left(\frac{\mathrm{I}}{\nu_{0}+n_{1}+\mathrm{I}}\right)$ von $\phi\left(\frac{\mathrm{I}}{v_{0}+n_{1}+\mathrm{I}}\right)$ erhält man durch Einsetzen in die Funktion $\phi(z)$ unmittelbar die folgende Abschätzung

$$
\left|\phi^{(\tau)}\left(\frac{\mathrm{I}}{v_{0}+n_{1}+\mathrm{I}}\right)\right|=O\left(e^{(2 s+6) n_{1} \log \frac{1}{n_{1}}}\right) \quad(\tau=2, \ldots, \sigma ; \sigma \leq s) .
$$

Damit ist für die Norm von $Q_{\phi}\left(\frac{\mathrm{I}}{\nu_{0}+n_{1}+\mathrm{I}}\right)$ als Funktion von $n_{1}$ die asymptotische Schranke

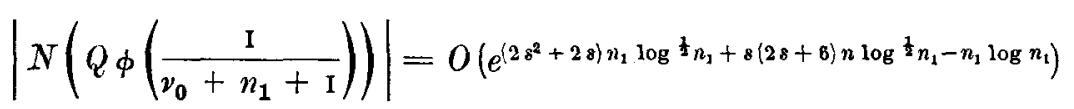

gefunden, also

$$
\left|N\left(Q \phi\left(\frac{\mathrm{I}}{v_{0}+n_{1}+\mathrm{I}}\right)\right)\right|<\mathrm{I}
$$

für alle $n_{1}$, die grösser als ein hinreichend grosses $n_{0}$ sind.

Es sei nun $n>n_{0}$, dann gilt (7) für alle $n_{1} \geq n$. Dann folgt aber aus $(7)$

$$
\phi\left(\frac{\mathrm{I}}{v_{0}+n_{1}+\mathrm{I}}\right)=0
$$

durch vollständige Induktion für alle $n_{1}=0,1, \ldots$

Die Stelle $z=0$ ist damit Häufungspunkt von Nullstellen der in der $\mathrm{Um}$ gebung von $z=0$ auf einem festen Blatt ihrer Riemannschen Fläche regulären analytischen Funktion $\phi(z)$. Dann muss aber

$$
\phi(z) \equiv 0
$$

sein, und damit ist der Beweis, dass die Bedingungen a) und b) hinreichend sind, erbracht. 
Nun fehlt noch der Nachweis, dass auch bei Satz II dessen Bedingungen a) und b) hinreichend sind. In diesen Bedingungen sind offenbar die Bedingungen a) und b) von Satz I enthalten. Also wissen wir nach Satz I bereits, dass auch bei Satz II zwischen der zu untersuchenden Funktion $g(z)$ und $z$ eine algebraische Abhängigkeit besteht. Wir sind fertig, wenn wir noch gezeigt haben, dass $g(z)$ eine eindeutige Funktion von $z$ ist. Dieser Nachweis wird in gewisser Analogie zum soeben Bewiesenen wie folgt geführt.

Da $g(z)$ eine algebraische Funktion von $z$ ist, gilt, wie wir bewiesen haben, an Stelle von Bedingung b) sogar die bedeutend schärfere Bedingung

$$
\varlimsup_{\nu \rightarrow \infty} \frac{\log H\left(g\left(\frac{\mathrm{I}}{\nu}\right)\right)}{\log v}<t .
$$

Mit $g(z)$ ist auch $g\left(\frac{z}{m+\mu z}\right)$ bei $z=0$ für alle Werte $\mu$ regulär und endlichvieldeutig.

Man bilde nun die Funktion

$$
\phi(z)=\sum_{\lambda, \mu=0}^{l, m} c_{\lambda \mu} z^{\lambda} g\left(\frac{z}{m+\mu z}\right)
$$

mit unbestimmten Koeffizienten $c_{\lambda \mu}$, die dann bei $z=0$ ebenfalls regulär ist. Ich verwende im Folgenden die gleichen Buchstaben für die Bezeichnung von Begriffen, die denjenigen des Beweises von Satz I analog sind, ohne dass hier zwischen diesen Buchstaben die gleichen Relationen wie bisher zu gelten brauchen. Es gibt nun mit von $m$ unabhängigem $r$ eine Umgebung $|z|<r$, in der die sämtlichen Funktionen $g\left(\frac{z}{m+\mu z}\right)$ mit $\mu=0, \mathrm{I}, \ldots, m$ regulär sin $\alpha$. In dieser Umgebung ist dann auch $\phi(z)$ regulär.

Die Koeffizienten $c_{\lambda \mu}$ sollen nun derart gewählt werden, dass sie nicht sämtlich verschwinden, und $\phi(z)$ auf dem festen Blatt seiner Riemannschen Fläche für $z=\frac{\mathrm{I}}{v}, v$ ganzzahlig und gleich $v_{0}+\mathrm{1}, \ldots, v_{0}+n$ mit $v_{0}=\mathrm{I}+\left[\frac{2}{r}\right]$, verschwindet. Setzt $\operatorname{man} z=\frac{\mathrm{I}}{\nu}$ in $\phi(z)=0$ ein, und denkt man sich für $g\left(\frac{\mathrm{I}}{\nu}\right)$ den Funktionswert auf dem festen Blatt seiner Riemannschen Fläche eingesetzt. so erhält man eine lineare homogene Gleichung für die $c_{\lambda \mu}$ mit Koeffizienten, die Produkte einer Potenz von $\frac{\mathrm{I}}{\nu}$ mit 


$$
g\left(\frac{\frac{\mathrm{I}}{\nu}}{m+\frac{\mu}{\nu}}\right)=g\left(\frac{\mathrm{I}}{m \nu+\mu}\right)
$$

sind. Diese letzteren Ausdrücke sind nach Voraussetzung algebraische Zahlen in einem festen Zahlkörper vom $s$-ten Grade. Jede solche lineare Gleichung zerfällt durch die Basisdarstellung der algebraischen Koeffizienten, die alle in diesem festen Zahlkörper liegen, in höchstens $s$ lineare homogene Gleichungen mit rationalen Koeffizienten, da wir uns wieder für ganze rationalen Lösungen $c_{\lambda \mu}$ interessieren wollen. Für die Zähler und den Hauptnenner dieser rationalen Koeffizienten erhalten wir wegen (8) die Abschätzung, dass ihre absoluten Beträge gleich $O\left(\nu^{l}((\nu+1) m)^{t(m+1)}\right)$ sind. Die ganzrationalen Koeffizienten des so aus den Forderungen, dass $\phi(z)=\mathrm{o}$ für $z=\frac{\mathrm{I}}{\nu_{0}+\mathrm{I}}, \ldots, z=\frac{\mathrm{I}}{v_{0}+n}$ verschwinde, entspringenden homogenen Gleichungssystems von höchstens $s \cdot n$ Gleichungen sind absolut genommen höchstens von der Grössenordnung

$$
O\left(\left(\nu_{0}+n\right)^{2 l}\left(\left(\nu_{0}+n+\mathrm{I}\right) m\right)^{2 t(m+1)}\right) .
$$

Es sei $(l+\mathrm{I})(m+\mathrm{I})$ die Anzahl der Unbestimmten, $\geq 2 s n$, und in $\mathbf{A b}$ weichung zum Beweis von Satz I werde nun

$$
l=\left[2 s n^{\frac{1}{1}}\right], \quad m=\left[1^{\frac{1}{2}}\right]
$$

gesetzt. Damit wird die Grössenordnung der ganzrationalen Koeffizienten in den linearen Gleichungen als Funktion von $n$ gleich

$$
O\left(e^{(4 s+3 t+1) n \log n}\right)
$$

und es ergeben sich mittels des Schubfachschlusses die nicht sämtlich verschwindenden, ganzen rationalen Grössen $c_{\lambda \mu}$ mit

$$
\left|c_{\lambda \mu}\right|=O\left(e^{(4 s+3 t+1) n^{\frac{1}{t}} \log n}\right) .
$$

Nun wird wie bei Satz I weiterverfahren, d.h. man zeigt wieder: Verschwindet $\phi(z)$ für $z=\frac{I}{v_{0}+1}, \cdots, z=\frac{I}{v_{0}+n_{1}}$ auf einem festen Blatt der Riemannschen Fläche von $g(z)$, so auch für $z=\frac{I}{\nu_{0}+n_{1}+1}$ auf dem gleichen Blatt, wenn $n_{1} \geq n$ und $n$ hinreichend gross ist. Daraus folgt nach dem Maximum- 
prinzip auch hier die Formel (6), und mittels derselben erhält man die gesuchte Abschätzung $\left|\phi\left(\frac{1}{v_{0}+n_{1}+I}\right)\right|$. Mit der gleichen Bestimmung von $\Gamma$ wie früher folgt

$$
\operatorname{Max}_{|z| \leq R}\left|\frac{\phi(z)}{\left(z-\frac{1}{v_{0}+1}\right) \cdots\left(z-\frac{\mathrm{I}}{v_{0}+n}\right)}\right|=O\left(e^{(4 s+3 t+1) n_{1}^{\frac{k}{1} \log n_{1}} \cdot l^{2} \cdot\left(\frac{r}{4}\right)^{-n_{1}}}\right)=O\left(\gamma_{3}^{n_{1}}\right)
$$

wobei $\gamma_{3}$, wie auch die folgenden $\gamma_{4}, \gamma_{5}, \ldots$ unabhängig von $n_{1}$ sind. Nach Formel (6) ergibt sich dann

$$
\left|\phi\left(\frac{\mathrm{I}}{v_{0}+n+\mathrm{I}}\right)\right|=O\left(\left(\frac{\gamma_{4}}{n_{1}}\right)^{n_{1}}\right) .
$$

Für den ganzrationalen Nenner $Q$ von $\phi\left(\frac{1}{v_{0}+n_{1}+1}\right)$ gilt

$$
|Q|=O\left(e^{\left.\left(28+\frac{3}{2} t+1\right) n_{1}^{\frac{1}{2} \log n_{1}}\right)}\right.
$$

und für die Konjugierten von $\phi\left(\frac{I}{\nu_{0}+\frac{I}{n_{1}+I}}\right)$

$$
\left|\phi^{(\tau)}\left(\frac{1}{v_{0}+n_{1}+1}\right)\right|=O\left(e^{(4 s+3 t+2) n_{1}^{\frac{1}{1}} \log n_{1}}\right) \quad(\tau=2, \ldots, \sigma ; \sigma \leq \kappa) .
$$

Damit hat man die Abschätzung gewonnen:

$$
\left|N\left(Q_{\phi}\left(\frac{1}{v_{0}+n_{1}+1}\right)\right)\right|=O\left(e^{\left.\left(2 s^{2}+\frac{3}{2} t s+s\right) n_{1}^{t} \log n_{1}+(s-1)(1 s+3 t+2) n_{1}^{t} \log n_{1}+\gamma_{6} n_{1}-n_{1} \log n_{1}\right)}\right.
$$

oder

$$
\left|N\left(Q \phi\left(\frac{\mathrm{I}}{\nu_{0}+n_{1}+\mathrm{I}}\right)\right)\right|<\mathrm{I}
$$

für alle $n_{1}$ mit $n_{1}>n_{0}$, also bei $n>n_{0}$ für alle $n_{1} \geq n$. Wieder ist also

$$
\phi\left(\frac{1}{\nu_{0}+n_{1}+1}\right)=0 \text {. }
$$

für alle $n_{1} \geq 0$, und daraus folgt auch dieses Mal das identische Verschwinden von $\phi(z)$ in $z$.

Führt man in (9) für $z=\frac{\mathrm{I}}{w}$ ein, und benennt man $g(z)$ mit $\psi\left(\frac{1}{z}\right)$, so ist

$$
g\left(\frac{z}{m+\mu z}\right)=g\left(\frac{1}{w m+\mu}\right)=\psi(m w+\mu)
$$


und aus (9) .wird nun

$$
\sum_{\lambda, \mu}^{l, m} c_{\lambda \mu} w^{-\lambda} \psi(m w+\mu)=0
$$

das heisst, $\psi(m w)$ genügt einer linearen homogenen Differenzengleichung mit Koeffizienten, die eindeutige Funktionen in $w$ sind. Dann muss aber die Lösung $\psi(m w)$ ebenfalls eine eindeutige Funktion von $w \operatorname{sein}^{1}$. Damit ist auch $g\left(\frac{1}{v}\right)$ eine eindeutige Funktion von $w$ und folglich ebenfalls eine eindeutige Funktion von $z$, was zu beweisen war.

Aus dem Bewiesenen geht unter anderem hervor, dass für jede algebraische, aber nicht rationale Funktion $g(z)$ die Funktionswerte $g\left(\frac{I}{\nu}\right)$ für alle $\nu=\nu_{0}+\mathrm{I}$, $v_{0}+2, \ldots$ nicht sämtlich in endlich vielen algebraischen Zahlkörpern liegen können. Es wäre sicher von Interesse, zu wissen, in welchem Umfange analoge Aussagen gelten, wenn die Folge $\left(\frac{I}{v}\right)$ durch andere Folgen ersetzt wird. Diese Frage, die mit der Frage nach Kriterien, die sich auf andere Folgen stützen, und deren Existenz eingangs erwähnt worden war, zusammenhängt, soll hier nicht behandelt werden. Ich möchte jedoch darauf hinweisen, dass das soeben angegebene Resultat über die Funktionswerte einer algebraischen Funktion an den Stellen der Folge $\left(\frac{I}{v}\right)$ noch wie folgt verschärft werden kann.

Bei den Sätzen I und II ist bemerkenswert, dass die Forderung b) viel schwächer ist als dieselbe tatsächlich erfüllt wird; das soll heissen, es wird nur gefordert

$$
\varlimsup_{\nu \rightarrow \infty} \frac{\log \log H\left(g\left(\frac{\mathrm{I}}{\nu}\right)\right)}{\log \nu}<\mathrm{I}
$$

und es stellt sich heraus, dass dann zusammen mit Ford rung a) stets

eintritt.

$$
\varlimsup_{\nu \rightarrow \infty} \frac{\log H\left(g\left(\frac{\mathrm{I}}{v}\right)\right)}{\log v}<t
$$

${ }^{1}$ Siehe $z$ 13. N. E. NöruvNd, Vorlesungen über Differenzenrechnung, Springer Verlag, Berlin, 1924. S. 282 
Nun lassen sich analog zu den Sätzen I und II mit der gleichen Methode Kriterien für Algebraizität und Rationalität, die wir wieder nur für die spezielle Folge $\left(\frac{1}{v}\right)$ formulieren wollen, beweisen, die in Bezug auf die Höhe von $g\left(\frac{I}{\nu}\right)$ keinen Spielraum lassen, dafür aber einen mit $v$ wachsenden Grad $s$ der algebraischen Zahlen $g\left(\frac{I}{v}\right)$ gestatten. Diese Kriterien, die die Aussage über die Funktionswerte einer algebraischen Funktion $g(z)$ an den Stellen $\left(\frac{I}{v}\right)$ verschärfen, lauten:

Satz III (2. Kriterium für Algebraizität):

Die Funktion $g(z)$, (die bei $z=0$ regulär sei), ist dann und nur dann algebraisch, wenn

a) die Funktionswerte von $g(z)$ für $z=\frac{\mathrm{I}}{v}, v=v_{0}+\mathrm{I}, v_{0}+2, \ldots$ bei ganzzahligem, geeignetem $\nu_{0}$ auf einem festen Blatt der Riemannschen Fläche von $g(z)$ sämtlich algebraische Zahlen kleiner als $s_{v}$-ten Grades sind, wobei $s_{v}=o\left(v^{\frac{1}{2}}\right)$ ist, und

b)$$
\varlimsup_{\nu \rightarrow \infty} \frac{\log H\left(g\left(\frac{I}{\nu}\right)\right)}{\log v}<t
$$

gilt.

Satz IV (2. Kriterium für Rationalität):

$g(z)$, (das bei $z=0$ regulär sei), ist dann und nur dann rational, wenn

a) die Werte $g\left(\frac{\mathrm{I}}{v}\right)$ auf einem Blatt für $v=v_{0}+\mathrm{I}, \ldots, v_{0}+\omega, \ldots$ bei ganzzahligem, geeignetem $\nu_{0}$ und mit $\omega=\mathrm{I}, \ldots, n$ algebraische Zahlen eines Körpers $\mathfrak{K}$ rom Grade $s_{n}$ sind mit $s_{n}=o\left(n^{\frac{1}{2}}\right)$, und

b)

$$
\varlimsup_{\nu \rightarrow \infty} \frac{\log H\left(g\left(\frac{\mathrm{I}}{\nu}\right)\right)}{\log \nu}<t
$$

ist.

Die Beweise dieser Kriterien sind dem hier durchgeführten Beweis von Satz II, der von dem Erfülltsein der schärferen Bedingung b), wie sie in den Sätzen III und IV verlangt wird, Gebrauch macht, leicht nachzubilden. Man hätte Satz II auch ohne Benutzung dieser Verschärfung, nur unter Verwendung 
der Bedingung b) des Satzes II, zusammen mit der Bedingung a) ganz analog zum Beweis von Satz I zeigen können. Die Sätze I und III, bzw. II und IV stellen nur die beiden Extremfälle solcher Kriterien unter Zugrundelegung der speziellen Folge $\left(\frac{I}{\nu}\right)$ dar, neben denen sich leicht beliebig viele weitere Kriterien, die sich auf die gleiche Folge $\left(\frac{I}{v}\right)$ stützen, formulieren lassen. 